\title{
CoviD-19 Utilising video technology in the cardiac catheter lab and operating theatre during the COVID-19 pandemic
}

\author{
Authors: Rajdip Dulai, ${ }^{A}$ Stanislav Hadjivassilev, ${ }^{A}$ Rick A Veasey, ${ }^{B}$ Nikhil Patel ${ }^{B}$ and Stephen Furniss ${ }^{B}$
}

The COVID-19 pandemic has significantly altered working practices within hospitals. This includes surgical theatres and cardiac catheter laboratories. Here we describe how we have harnessed the use of video technology to improve the running of the cardiac catheter lab in our institution, reducing the exposure of staff members to COVID-19, reducing the need for personal protective equipment (PPE) and maintaining and enhancing educational and teaching opportunities for trainees.

KEYWORDS: COVID-19, telemedicine, video technology, cardiac cath lab

DOI: 10.7861/fhj.2020-0051

\section{The problem}

The cardiac catheter lab is used for many procedures requiring fluoroscopy, including angiograms, primary percutaneous coronary intervention (PCI) and pacemaker insertions. Eastbourne District General Hospital has two fully functional catheter labs. The smooth running of the catheter lab requires multiple staff members including physicians, registrars, nurses, physiologists and radiographers.

Although some elective procedures have been postponed during the COVID-19 pandemic, urgent and emergency procedures including pacemaker insertions, generator changes and primary PCI are still mandated. ${ }^{2,3}$

The COVID-19 pandemic has posed many challenges in the catheter lab, including the following:

> Many patients show signs of or have confirmed COVID-19 but still require an urgent procedure. This poses a risk to staff members and thus limiting the number of staff in the catheter lab is vitally important.

> Normally more than five staff members would be in a catheter lab, making it problematic to maintain distancing rules.

$>$ Operators are in close proximity to the patients (within 2 metres) performing potential aerosol-generating procedures

Authors: ${ }^{A}$ Cardiology registrar, Eastbourne District General Hospital, Eastbourne, UK; ${ }^{\mathrm{B}}$ consultant cardiologist, Eastbourne District General Hospital, Eastbourne, UK and thus procedures should be limited to only one operator. This poses the additional challenge of meeting the training needs of registrars.

> In many cases a second opinion from another physician is needed. Normally this would require the second physician to go into the lab to review images or to give advice, again making it harder to maintain distancing and increasing exposure for staff and patients.

\section{A solution}

We sought to reduce the number of staff members in the catheter lab in line with current guidance while at the same time maintaining patient safety and retaining a learning platform for junior doctors and allied health care professionals.

We set up a video conferencing facility within the catheter lab, which allowed communication to healthcare providers outside the lab. This involved two small web cameras mounted in the catheter lab. Also, the fluoroscopy images and diagnostic recordings such as the ECG were anonymised and streamed into a custom-made software (Lifestream) to allow simultaneous viewing of all stages of the procedure (Fig 1).

Initially the live video was streamed over the local network to a nurse outside the catheter lab, allowing the nurse to see exactly what was happening in the lab in real time. The system also allowed communication to enable the nurse to provide equipment

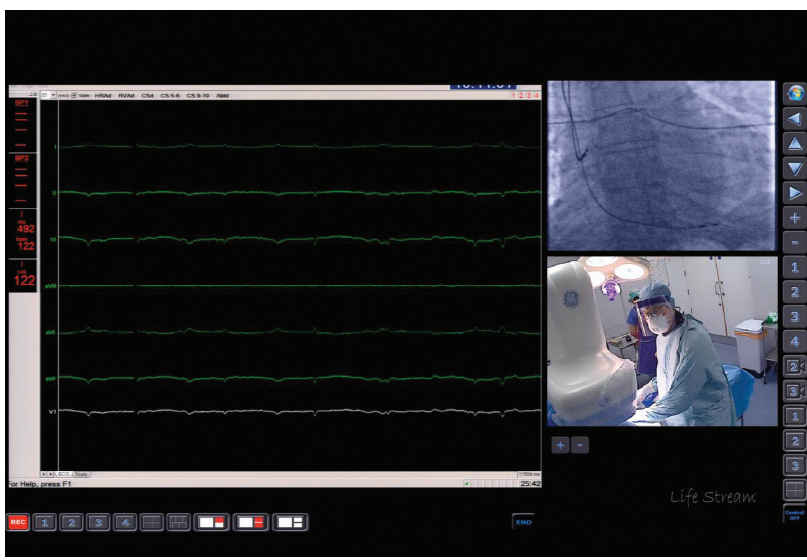

Fig 1. Lifestream interface, combining live video streaming of the procedure together with diagnostic recordings and images. 
to the catheter lab without going inside, thus reducing exposure to COVID-19. In some cases this was extended to streaming in real time to a consultant colleague, allowing effective remote proctoring and the provision of a second opinion.

\section{Discussion}

The use of video streaming in the catheter lab has helped us to fulfil our main aim of reducing the number of staff and allied healthcare professionals in the catheter lab, enabling adherence to distancing guidelines and reducing exposure to COVID-19. This has also reduced the need for PPE, which is in high demand.

The system has also allowed us to stream procedures remotely to consultant colleagues outside the catheter lab. This has been particularly advantageous as many cases require input from two or more consultants, for example when deciding whether a patient should go on to have cardiac surgery or a stent insertion.

One of the challenges of the COVID-19 pandemic faced by many procedure-based specialties is how to provide learning experiences to trainees. The system has allowed consultants to proctor cases remotely, thus providing support and confidence to trainees. ${ }^{4}$ The system has also been set up on a dedicated server, enabling all cases to be recorded. This provides a valuable teaching aid and may potentially be used for auditing or root cause analysis investigations in the future. The system may be used in any procedural setting including operating theatres and endoscopy units.

The COVID-19 pandemic has accelerated the use of telemedicine by healthcare professionals. The use of applications such as Zoom or Microsoft Teams has exponentially increased, replacing face-toface meetings and clinic appointments. ${ }^{4}$ The system described can be implemented by transferring the video graphics array output from the cameras, fluoroscopy screens and ECG monitoring to a single computer which has screen sharing capability in a video call.

The uptake of telemedicine has been slower in situations in which there is patient identifiable information. This barrier was overcome by using the Lifestream system to crop patient-identifiable information in the streaming feed, thus anonymising all data and making the system GDPR-compliant. ${ }^{5}$ As a result, with patient consent, the stream may be shared in a video call with colleagues remotely within other applications such as Microsoft Teams.

The use of robotic techniques has also been advocated and used during the COVID-19 pandemic, for example for gynaecological procedures. ${ }^{6}$ This has the advantage of also protecting the primary operator. However robotic systems are not available in all hospitals and their high cost prevents rapid uptake; for example, the CorPath GRX system used for robotic PCI costs in excess of $\$ 650,000$.?

\section{Conclusion}

The described video streaming system has allowed the department to effectively manage COVID-19 patients in the catheter lab without compromising patient safety. In addition, it has reduced staff exposure and provided a valuable learning tool. The COVID-19 pandemic has accelerated our use of video technology, which will have benefits in the future, allowing remote proctoring and multidisciplinary team meetings to occur remotely without delay.

\section{Funding}

Rajdip Dulai is funded by an unrestricted research grant from Eastbourne Cardiology Research Charity.

\section{References}

1 Kern M. How many people do you need to do a cardiac cath? Cath Lab Digest, 11 February 2010. Available from www.cathlabdigest. com/articles/How-Many-People-Do-You-Need-Do-a-Cardiac-Cath.

2 Szerlip M, Anwaruddin S, Aronow HD et al. Considerations for cardiac catheterization laboratory procedures during the COVID-19 pandemic: perspectives from the Society for Cardiovascular Angiography and Interventions Emerging Leader Mentorship (SCAI ELM) members and graduates. Catheter Cardiovasc Interv 2020, in press (doi: 10.1002/ccd.28887).

3 NHS England. Clinical guide for the management of cardiology patients during the coronavirus pandemic. NHSE, 2020. Available from www.england.nhs.uk/coronavirus/wp-content/uploads/sites/ 52/2020/03/specialty-guide-cardiolgy-coronavirus-v1-20-march. pdf [Accessed 24 April 2020].

4 NHS Digital. Messaging tool for NHS to support remote working during coronavirus outbreak. NHS Digital, 2020. Available from https:// digital.nhs.uk/news-and-events/latest-news/messaging-tool-for-nhs-tosupport-remote-working-during-coronavirus-outbreak [Accessed 24 April 2020].

5 Schütze B, Kämmerer M. [Legally regulated teleradiology: implementation of data protection legal requirements.] Radiologe 2019;59:637-42.

6 Kimmig R, Verheijen RHM, Rudnicki M. Robot assisted surgery during the COVID-19 pandemic, especially for gynecological cancer: a statement of the Society of European Robotic Gynaecological Surgery (SERGS). J Gynecol Oncol 2020;31:e59.

7 Beck DL. The robot will see you now... robotics in the cath lab have staff breathing a sigh of relief. Cardiology Interventions, 3 August 2017. Available from www.acc.org/latest-in-cardiology/ articles/2017/08/01/18/42/the-robot-will-see-you-now-robotics-inthe-cath-lab-have-staff-breathing-a-sigh-of-relief [Accessed 8 May 2020].

Address for correspondence: Dr Rajdip Dulai, Cardiology Research Department, East Sussex Hospitals NHS Trust, Eastbourne District General Hospital, Kings Drive, Eastbourne, Sussex BN21 2UD, UK.

Email: rajdip.dulai@nhs.net 